\title{
Frontières
}

\section{Accompagner le deuil périnatal}

\section{Suzy Fréchette-Piperni}

Volume 16, numéro 2, printemps 2004

Deuil, blessure vive

URI : https://id.erudit.org/iderudit/1074118ar

DOI : https://doi.org/10.7202/1074118ar

Aller au sommaire du numéro

Éditeur(s)

Université du Québec à Montréal

ISSN

1180-3479 (imprimé)

1916-0976 (numérique)

Découvrir la revue

\section{Citer cet article}

Fréchette-Piperni, S. (2004). Accompagner le deuil périnatal. Frontières, 16(2), 58-63. https://doi.org/10.7202/1074118ar

\section{Résumé de l'article}

Le deuil périnatal est un deuil complexe à cause des multiples implications pour les parents. L'impact de ce deuil est mal compris et minimisé par l'entourage qui ne donne pas le soutien adéquat. Des infirmières du Centre hospitalier Pierre-Boucher à Longueuil assurent le suivi de deuil périnatal depuis 1991 et ont accompagné plus de 450 familles. Un accompagnement professionnel brise l'isolement des parents et leur permet d'exprimer leur souffrance favorisant une progression saine du deuil. Cet accompagnement améliore la compréhension dans le couple et diminue les répercussions négatives sur la famille. Il a avantage à se continuer durant la grossesse suivante.
Ce document est protégé par la loi sur le droit d'auteur. L'utilisation des services d'Érudit (y compris la reproduction) est assujettie à sa politique d'utilisation que vous pouvez consulter en ligne.

https://apropos.erudit.org/fr/usagers/politique-dutilisation/ 


\section{Résumé}

Le deuil périnatal est un deuil complexe à cause des multiples implications pour les parents. L'impact de ce deuil est mal compris et minimisé par l'entourage qui ne donne pas le soutien adéquat. Des infirmières du Centre hospitalier PierreBoucher à Longueuil assurent le suivi de deuil périnatal depuis 1991 et ont accompagné plus de 450 familles. Un accompagnement professionnel brise l'isolement des parents et leur permet d'exprimer leur souffrance favorisant une progression saine du deuil. Cet accompagnement améliore la compréhension dans le couple et diminue les répercussions négatives sur la famille. Il a avantage à se continuer durant la grossesse suivante.

Mots clés : deuil périnatal - père - mère accompagnement.

\section{Abstract}

Perinatal grief is very intricate and complex because of the various implications for the parents. The impact of perinatal grief is not well understood by relatives and often played down. Some nurses of the Centre hospitalier Pierre-Boucher de Longueuil have been providing perinatal mourning follow-up since 1991 and have helped more than 450 families. A professional follow-up breaks the parents' loneliness and gives them manage opportunity to express their suffering in a positive way, thereby helping them get through their grief. The follow-up improves the dialogue and understanding within the couple and reduces the negative repercussions on family members. The professional help should also be continued during the next pregnancy.

Key words: perinatal mourning - fathermother-monitoring.

\section{ACCOMPAGNER LE DEUIL PÉRINATAL}

\author{
Suzy Fréchette-Piperni, B.Sc., \\ infirmière spécialisée en deuil périnatal \\ Centre hospitalier Pierre-Boucher, Longueuil.
}

Même si aujourd'hui plus d'intervenants sont conscients que la perte d'un bébé, durant la grossesse ou dans les premières semaines de vie, entraîne un vrai deuil, beaucoup ignorent à quel point ce deuil est différent des autres et est souvent plus douloureux et plus difficile à faire que celui d'un adulte aimé. L'entourage des parents sousestime aussi l'impact de la perte d'un bébé sur la famille et, par conséquent, les parents ne reçoivent pas le soutien qui les aiderait à traverser cette épreuve. Un accompagnement professionnel diminue les risques que ce deuil difficile entraîne des perturbations importantes chez les parents et dans la famille. Une équipe d'infirmières, spécialement formées, du Centre hospitalier Pierre-Boucher de Longueuil, offre cet accompagnement aux parents endeuillés. Les parents bénéficient d'un cadre réconfortant pour exprimer leurs émotions et partager, avec quelqu'un disponible pour les aider, les différentes problématiques qu'ils rencontrent dans leur deuil. Ils reçoivent du soutien pour traverser les difficultés que la perte du bébé entraîne dans leur couple et sont guidés dans l'approche à la fratrie.
Ce soutien se poursuit durant la grossesse suivante et au besoin après la naissance du nouveau bébé.

Selon Rousseau (1997), le deuil périnatal est un deuil complexe, différent de tous les autres deuils à cause des multiples implications de cette mort pour les parents. Quand un enfant de quelques années est perdu, il est clair que les parents pleurent leur enfant. Mais lors d'un deuil périnatal, ce n'est pas aussi évident. Ce n'est pas toujours la perte du bébé qui fait le plus mal. Lors du suivi de deuil, on peut remarquer que certains parents vont parler beaucoup du bébé perdu, mais que d'autres parleront surtout d'autres choses. Certains parents pleurent un objet d'amour, le petit Samuel ou la petite Fanny, et pour ces parents, leur deuil ressemblera plus à celui vécu par des parents qui ont perdu un enfant plus vieux. Plusieurs ont de la peine de ne pas pouvoir être parents, car ils se sentaient prêts à franchir cette étape, importante dans leur processus de maturation (Dohmen, 1996). Les parents qui n'ont pas d'autres enfants vivants sont particulièrement touchés.

Certains parents peuvent être très affectés par la diminution d'estime de soi consécutive à la perte du bébé. Donner naissance à un bébé en santé réaffirme la féminité de 
la mère et la virilité du père. La mort du bébé est souvent vécue comme une grande blessure narcissique et entraîne un sentiment d'échec. Les parents peuvent se sentir honteux, diminués aux yeux des autres. Ils n'ont pas réussi à mettre au monde un enfant vivant, ce que, pourtant, les autres parents réussissent à faire.

Des deuils antérieurs non résolus peuvent compliquer le deuil, par exemple: la perte d'un parent durant l'enfance (Léon, 1992) ou une perte périnatale antérieure comme, par exemple, un avortement. Ces deuils refont surface ou viennent interférer avec la résolution du deuil actuel. On voit souvent ressortir les difficultés de la mère dans sa relation avec sa propre mère. C'est qu'au moment où la femme a tellement mal d'avoir perdu son bébé, elle se tourne spontanément vers sa mère pour être consolée et soutenue. Si la grand-mère du bébé ne donne pas le soutien espéré, la mère réalise souvent que ce soutien lui manque depuis l'enfance. Ceci fait remonter toute la peine et la colère reliées au sentiment de perte de la bonne mère qu'elle n'a jamais eue (Bydlowski, 1997). C'est vrai aussi pour le jeune père.

Un autre deuil associé peut augmenter l'impact du présent deuil, comme par exemple, le conjoint qui ne veut plus d'autres enfants ou la grossesse de la dernière chance, dans les cas d'infertilité. La mère devra, en plus du deuil du bébé décédé, faire le deuil des enfants futurs qu'elle n'aura pas et du bonheur d'être enceinte et d'allaiter (Fréchette-Piperni, 1999). De plus, une grossesse joue souvent un rôle complémentaire à celui de mettre un bébé au monde, comme de resserrer les liens entre les conjoints ou tout simplement le retenir, faire plaisir à ses parents ou leur prouver qu'on est capable d'être responsable, réparer sa propre image comme mère à cause d'une expérience de maternité antérieure négative, etc. Le rôle que cette grossesse venait jouer, en étant contrarié, augmente l'impact de la perte en ajoutant une autre perte qui amplifie la détresse actuelle. La grossesse joue aussi toujours un rôle inconscient mais crucial chez la mère (Bydlowski, 1997).

\section{LA NÉCESSITÉ DU SUIVI DE DEUIL}

Le soutien dans les hôpitaux, pour les parents qui perdent un bébé, s'est beaucoup amélioré ces dernières années, surtout pour les parents qui vivent une perte en fin de grossesse. Le plus souvent, les parents sont entourés par le personnel, peuvent partager leur peine, exprimer leurs émotions et ils sont écoutés. Ils sont guidés dans les décisions à prendre et soutenus.

Mais le retour à la maison est très difficile : c'est souvent à ce moment-là que les parents réalisent pleinement l'étendue de leur perte: la belle chambre qui attend le bébé, le ventre vide, les bras vides, la pensée qu'ils ne pourront plus jamais ni voir, ni prendre leur bébé. Tous leurs rêves se sont effondrés, ils se sentent abandonnés, laissés à eux-mêmes pour faire face à ce deuil, souvent le premier deuil important de leur vie. Ils se sentent devant un chemin difficile, sans balises ni soutien. D'où leur question: "Comment allons-nous faire pour passer à travers cette épreuve?»

Cela ne fait pas beaucoup d'années qu'on parle de suivi ou d'accompagnement de deuil périnatal. Il n'est pas question, dans cet article, de thérapie de deuil mais plutôt d'accompagnement du deuil. Même si le deuil est associé à une période de souffrance intense, les parents n'ont pas nécessairement besoin du soutien d'un thérapeute spécialisé en thérapie de deuil, dont les services s'adressent plutôt aux parents qui sont bloqués dans leur deuil ou qui vivent un deuil pathologique. Il est entendu que certaines personnes particulièrement à risques, comme celles souffrant de troubles psychiatriques avant la perte, doivent être d'emblée référées à des professionnels spécialisés.

Pour la majorité des parents, le seul fait d'être accompagnés par quelqu'un qui connaît le deuil périnatal peut faire une différence importante. Par ailleurs, plusieurs recherches rapportées par Stroebe et al. (2001) réfutent le bien-fondé des interventions systématiques après un deuil, soutenant que celles-ci sont inefficaces et même dommageables dans certains cas. Par contre, les auteurs s'entendent sur le fait que, dans des cas présentant des facteurs de risque, des interventions précoces peuvent être bénéfiques pourvu qu'elles soient offertes et non pas imposées.

Les pertes périnatales comportent plusieurs facteurs de risque (Rousseau, 1988) comme :

- Une mort imprévue: les parents attendent un événement heureux et non pas la perte du bébé. D'une minute à l'autre, leur vie bascule et leurs rêves s'envolent.

- Des circonstances souvent traumatisantes, par exemple: une hémorragie lors d'une fausse couche ou une césarienne d'urgence, où la mère a pu se sentir en danger de mort. On peut penser aussi au traumatisme des parents qui doivent prendre la décision de mettre fin à la vie du bébé qu'ils aiment, parce que celui-ci est porteur de malformations graves, qu'il serait non viable à la naissance ou qu'il aurait une qualité de vie grandement compromise. Dans certains cas, des symptômes de stress post-traumatique sont encore présents après plusieurs mois.
- Un pauvre soutien social : le soutien social est proportionnel à la reconnaissance de l'impact de la perte du bébé pour les parents. Dans notre société, cette perte est banalisée et l'impact est sous-estimé, surtout si la perte se produit en début de grossesse. Il est aussi répandu de penser que les adolescentes, les femmes qui n'ont pas de conjoint, qui n'avaient pas planifié ou désiré être enceinte, n'ont pas de peine et n'auront pas besoin de soutien émotionnel.

Pourtant, ce n'est pas l'âge de la grossesse ni les circonstances de la conception ou de la perte qui déterminent le chagrin qui va suivre mais plutôt la nature et l'intensité des liens déjà formés avec le bébé et la signification de cette grossesse pour la femme et son conjoint. Plusieurs autres éléments auront aussi un impact sur le deuil des parents, comme leur perception de l'événement et leurs ressources personnelles. On peut penser ici à la notion de hardiness, cette capacité à faire face positivement aux difficultés de la vie (voir Lang, 2002).

\section{L'EXPÉRIENCE DU CENTRE HOSPITALIER PIERRE-BOUCHER À LONGUEUIL}

Depuis 1991, une équipe d'infirmières du service de natalité, spécialement formées, assurent l'accompagnement de deuil des parents qui perdent un bébé. Le service a été mis sur pied pour répondre à la demande répétée des parents en deuil.

Le service est offert aux parents, durant l'hospitalisation. Un premier contact peut être fait durant le séjour hospitalier ou par téléphone après le retour à la maison. Par la suite, les contacts, surtout téléphoniques, mais parfois en face à face, sont ajustés selon le besoin des parents. Le suivi peut être très court, un ou deux contacts, ou s'étendre sur quelques années, notamment si les parents vivent une autre perte ou ont besoin de soutien durant une grossesse subséquente. Les parents ont le numéro d'une boîte vocale où ils peuvent laisser un message s'ils ont besoin de parler à l'infirmière entre les appels. Si les parents semblent en détresse, l'infirmière communique avec eux régulièrement. Quand ils semblent aller mieux, ils communiquent avec l'infirmière, selon leurs besoins.

Le suivi de deuil est disponible autant pour les pertes en fin de grossesse ou en période néonatale que pour les pertes précoces comme les fausses couches, les grossesses ectopiques ou les interruptions médicales de grossesse pour anomalies. Dans ces derniers cas, le suivi de deuil est disponible à la demande des parents ou à la suite d'une référence d'une infirmière ou d'un médecin. 
Le service est disponible sans égard à la date de la perte. Il n'est pas rare que des mères nous rappellent, après avoir refusé ou écourté un suivi, pour avoir du soutien des mois ou même des années plus tard. Depuis le début de ce service, les infirmières de l'équipe ont accompagné plus de 450 familles.

\section{AVANTAGES DE L'ACCOMPAGNEMENT}

Le suivi de deuil permet de briser l'isolement des parents (Fréchette-Piperni, 1999). C'est sécurisant et réconfortant pour les parents de savoir qu'ils ne sont pas seuls pour traverser cette épreuve, qu'ils ont une personne-ressource vers qui se tourner, s'ils ne se sentent pas bien.

Le soutien de l'entourage, quand il y en a, est souvent limité dans le temps, se résumant aux premiers jours ou aux premières semaines: les parents, selon les proches, doivent s'en remettre rapidement. L'aide professionnelle est d'autant plus appréciée qu'elle est disponible quand le soutien de l'entourage a disparu. Le soutien est offert autant au père qu'à la mère. Le père est le grand oublié après une perte périnatale, le focus étant mis sur le chagrin de la mère. Pourtant, lui aussi a perdu son bébé et a besoin de soutien.

Devant le peu de compréhension de l'entourage, les parents peuvent avoir tendance à se replier sur eux-mêmes. On peut penser à la maman qui fait une fausse couche et qui se fait dire: "Une fausse couche, ce n'est pas grave» et qui se sent jugée, comme anormale parce qu'elle vit un immense chagrin. Le suivi de deuil permet à la souffrance de s'exprimer pour que le deuil puisse évoluer plus rapidement et plus sainement. Les parents peuvent exprimer des émotions qui, pour eux, sont presque indicibles, sans se sentir jugés. Parce que ces émotions sont «normalisées » dans le cadre de la relation avec l'infirmière, elles deviennent moins bouleversantes pour les parents : elles se transforment et prennent moins d'importance. Parler avec l'infirmière permet aussi d'aider à mettre des mots sur des émotions confuses, difficiles à expliquer. Les émotions se clarifient et la personne évolue plus facilement vers une certaine sérénité. La «normalisation » du processus de deuil des parents, par l'intervenant, fait contrepoids aux remarques de l'entourage selon lesquelles les parents devraient avoir déjà passé au travers de leur deuil, que ce dernier n'est pas si important puisqu'ils n’ont pas vraiment connu le bébé. Parfois, les grands-parents ou d'autres proches sont très éprouvés par le mort du bébé et ont aussi besoin du soutien de l'infirmière. $\mathrm{Si}$ eux aussi sont soutenus, ils seront plus disponibles pour donner du soutien aux parents.

\section{EXEMPLES DE SUJETS QUI PEUVENT ÊTRE ABORDÉS LORS D'UN SUIVI DE DEUIL}

Certains parents partagent facilement les émotions qui les habitent et l'infirmière n'a qu'à être à l'écoute de leur expérience. Pour d'autres parents, il sera plus facile d'aborder, dans un premier temps, des sujets moins menaçants pour les aider à évoluer dans leur deuil. Ces points doivent être proches de leurs préoccupations. Voici quelques exemples des points qui peuvent être abordés lors d'un accompagnement de deuil :

\section{LES PERTES ANTÉRIEURES}

- Les personnes significatives décédées: les circonstances des pertes et comment le deuil a été vécu. Les pertes antérieures non résolues nécessitent, en général, une référence en psychothérapie.

- Les pertes périnatales antérieures des parents: une fausse couche antérieure ou une IVG gardée secrète peut avoir occasionné un deuil non résolu qu'il est possible de faire évoluer, dans le cadre du suivi de deuil.

- Les pertes périnatales dans la famille:

1. les frères et les sœurs du couple, qui sont décédés alors qu'ils étaient bébés. Le sujet est tabou dans plusieurs familles. On peut faire ressortir les effets pervers du silence et faire le parallèle avec nos interventions d'aujourd'hui.

2. les bébés décédés récemment dans la famille. Si c'est un sujet évité, il sera difficile pour les parents de partager leur peine. Cela permet aussi de déceler parmi les proches qui pourrait être un bon soutien pour les parents.

\section{LES PROBLĖMES CONCOMITANTS À LA CRISE}

- Mentionnons, par exemple, que si le grand-père se meurt d'un cancer, la mort du bébé revêt moins d'importance pour l'entourage: il y aura moins de soutien disponible pour les parents.

\section{L'HISTOIRE}

\section{DE LA GROSSESSE ACTUELLE}

- Les difficultés à concevoir : y a-t-il eu difficulté de conception ou grossesse surprise? Les difficultés peuvent être mieux résolues si les émotions qui s'y rattachent sont exprimées.

- Les problèmes durant la grossesse: il arrive souvent que de la culpabilité y soit reliée, par exemple, s'il y a eu saignement en début de grossesse et que la mère soit retournée travailler au lieu de rester alitée durant neuf mois. On peut alors répondre aux questions restées en suspens et replacer les choses dans un contexte plus réaliste. Si la grossesse a été très difficile, la mère peut avoir vécu beaucoup d'ambivalence et penser que la perte du bébé est une punition.

\section{LE DÉROULEMENT}

DES ÉVÉNEMENTS

- L'annonce du diagnostic: en parler peut faire ressortir la colère reliée à une annonce dénuée de compassion.

- L'accouchement: il n'est pas facile pour les parents de partager leur expérience d'accouchement, car il finit mal. L'accouchement en lui-même est un événement spécial dans la vie d'un couple: inquiétudes, préparation, contractions, douleur, poussée, etc. Et ils ont besoin d'en parler.

- Le bébé : les parents dont le bébé est mort ont envie de parler de leur bébé tout comme ceux dont le bébé est vivant. Même s'il est mort, ce bébé a un poids, une longueur, des caractéristiques qui lui sont propres, comme les bébés vivants. Il peut avoir plus ou moins de cheveux et des ressemblances avec les parents ou les autres enfants. En posant des questions, cela montre l'importance qui est accordée à leur bébé et le rend plus réel. Cela augmente aussi l'estime de soi des parents qui réalisent qu'ils ont fait quelque chose de positif.

- Les souvenirs : les parents peuvent être heureux de montrer les photos de leur bébé. Souvent personne ne veut les voir autour d'eux ou les parents ont peur de traumatiser les gens et n'osent pas les montrer. Mais, en général, les parents trouvent leur bébé beau et sont fiers de le montrer. Les parents qui n'ont pas de souvenir comme dans le cas d'une fausse couche précoce peuvent être encouragés à utiliser quelque chose de tangible et de significatif pour rappeler que le bébé a existé. Par exemple, une maman a acheté un encadrement sur lequel il y a un petit ange et elle a mis leur photo de famille dedans.

- Les décisions relatives au bébé : revoir les décisions relatives au bébé (autopsie, funérailles, disposition du corps). Supporter les décisions des parents et suggérer des rituels, au besoin.

\section{LES SYMPTÔMES DE DEUIL}

Les parents qui perdent un bébé vivent un stress intense. Non seulement ils se sont sentis impuissants à empêcher la perte, mais ils peuvent se sentir tellement mal physiquement qu'ils ont peur de mourir ou 
tellement ébranlés émotionnellement qu'ils ont peur de devenir fous. Beaucoup de ces symptômes sont causés par l'anxiété (Lindemann, 1944). Nous explorons la présence de ceux-ci à chaque contact jusqu'à leur disparition, rassurons les parents sur leur normalité, ce qui favorise la diminution de symptômes et les conseillons au besoin.

- Les troubles du sommeil : l'insomnie, les cauchemars ou les rêves troublants sont fréquents.

- Les symptômes somatiques se manifestent souvent dans les premières semaines du deuil: diminution de la résistance aux infections, troubles gastro-intestinaux, faiblesse, fatigue, diminution d'énergie, migraines, troubles de l'appétit, etc.

- Les difficultés de fonctionnement dans la vie quotidienne ne sont pas rares: difficultés de concentration, pertes de mémoire, désorganisation, etc. Les pères qui retournent travailler rapidement ne sont pas toujours fonctionnels et productifs. Parfois, ils ne font pas le lien avec la perte qu'ils ont subie.

- Les hallucinations, comme de sentir bouger le bébé dans son ventre ou de l'entendre pleurer, ne sont pas rares chez la mère. Les hallucinations stressent énormément la mère qui a l'impression qu'elle est en train de devenir folle: les gens autour d'elle pensent aussi la même chose et sont très inquiets. Les parents ont besoin d'être rassurés sur la normalité de ces phénomènes (Fréchette-Piperni, 1999).

\section{LES ÉMOTIONS}

Les parents qui vivent un deuil périnatal vivent des émotions qui sont universelles comme la colère, la culpabilité, la tristesse, entre autres, mais celles-ci ont une connotation différente.

- La colère : la perte d'un bébé entraîne un grand sentiment d'injustice. Les parents vivent beaucoup de frustration de ne pas avoir ce bébé qu'ils désiraient alors que d'autres parents qui n'en veulent pas ont des bébés en bonne santé et les négligent ou les maltraitent. Ils peuvent aussi ressentir de la colère contre le médecin, l'hôpital, les proches, les autres femmes enceintes et même contre le bébé qu'ils aimaient tellement et qui leur fait si mal.

- La culpabilité est aussi une émotion envahissante, surtout chez la mère. Durant la grossesse, il y a une telle symbiose entre la mère et son bébé qu'elle se sent totalement responsable du bien-être de son bébé. Elle se sent coupable de tout: du verre de vin,

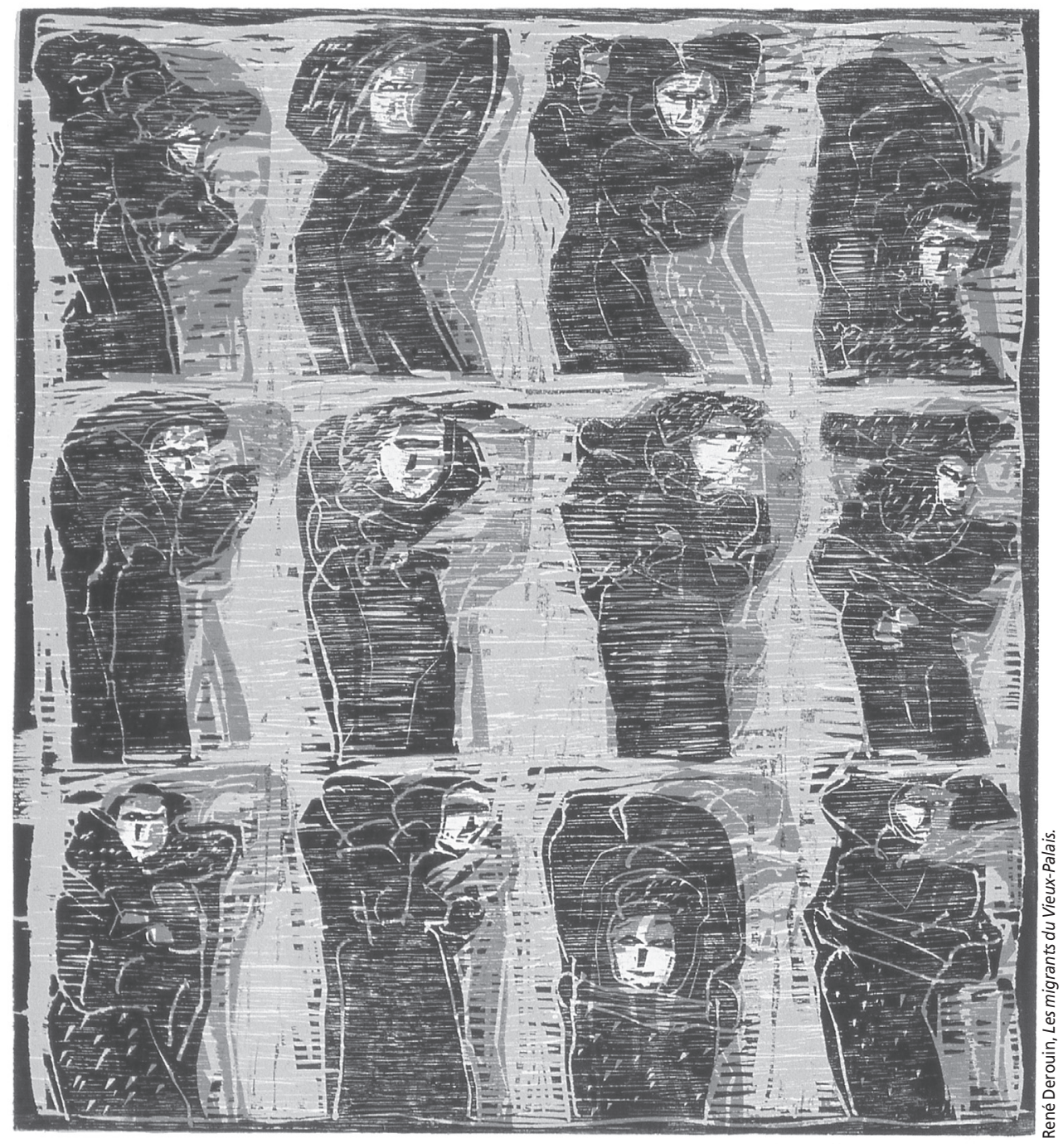

du stress du travail, de la relation sexuelle de la veille, etc. Elle peut se sentir punie pour une faute passée ou se sentir responsable de la peine du conjoint, des autres enfants et de l'entourage, etc.

- La tristesse et même une souffrance intense est rapportée par les parents. Même quand le chagrin des premières semaines commence à diminuer, il est ravivé par des événements comme la sortie des poussettes au printemps ou la naissance d'un autre bébé dans la famille, la fête des Mères et des Pères, ou le temps des Fêtes, etc. Dans certains cas, la tristesse persistera toujours, comme par exemple, le père qui perd un garçon et n'aura que des filles par la suite ou la mère qui se voyait vieillir entourée d'enfants, qui en perd plusieurs et en réchappe un seul.

\section{LES RELATIONS DE COUPLE}

Les parents sont souvent surpris des tensions qu'ils vivent comme couple après la perte. Les deux sont parfois sur la même longueur d'ondes les premiers jours, mais leurs réactions deviennent différentes par la suite.

- L'une des sources de difficulté, c'est que les parents assument souvent que parce qu'ils s'aiment et qu'ils ont perdu le même bébé, ils vivront tous les deux leur deuil de la même façon. Mais ce sont deux individus distincts qui sont en deuil, avec leur propre personnalité, leur histoire personnelle de deuil sans compter l'influence des facteurs familiaux et culturels. Chaque parent est attaché différemment au bébé, ce qui a une influence sur l'intensité du chagrin. L'impact prévu de l'arrivée du bébé dans leur vie est différent et la mère éprouve souvent plus de difficulté à se réorganiser après la mort de celui-ci.

- Chacun exprime son deuil différemment, par exemple, la mère peut pleurer davantage et le père se sentir plus irritable.

- Le deuil n'est pas vécu avec synchronisme dans le rythme. 
- Une autre source de difficultés découle des attentes élevées envers le conjoint. Or, il est souvent difficile de donner du soutien quand on a aussi mal que l'autre. D'où l'importance d'encourager les parents à trouver une autre source de soutien dans l'entourage.

- Il peut être difficile de communiquer leurs sentiments pour certains conjoints parce qu'ils ne l'ont jamais fait.

- Parfois l'un des conjoints veut empêcher l'autre de vivre son deuil à sa façon: "Arrête de pleurer, tu te fais du mal pour rien. » "C'est mieux que tu n'ailles pas au groupe de soutien, ça ne t'apportera rien de bon », ce qui augmente les tensions dans le couple.

- La reprise des relations sexuelles peut aussi être problématique, les deux conjoints n'étant pas au même diapason. Pour un des conjoints, les relations sexuelles peuvent être un moyen d'oublier sa peine, de se sentir vivant, mais l'autre peut se sentir coupable de ressentir du plaisir. Chez l'homme, la sexualité peut masquer un besoin de réconfort: il peut se sentir rejeté s'il subit un refus. L'infirmière peut expliquer les façons différentes pour chaque conjoint de vivre un deuil et les encourager à respecter la façon de réagir de l'autre. Beaucoup de conflits sont ainsi désamorcés dans le couple. La seule façon de survivre comme couple, c'est de réaliser et de respecter que l'autre vit son deuil différemment de soi.

\section{LES AUTRES ENFANTS}

Les réactions des autres enfants à la mort du bébé sont souvent la préoccupation majeure des parents. Ils aimeraient protéger leurs enfants de la souffrance, mais leur rôle comme parents est plutôt de se servir des événements de la vie pour enseigner à leurs enfants comment faire face aux difficultés (Fréchette-Piperni, 2001). L'infirmière guide les parents dans leur approche auprès des enfants et les renseigne sur la meilleure attitude pour diminuer les risques de perturbations.

\section{LES RESSOURCES}

Les parents sont encouragés à utiliser les ressources du milieu, par exemple, en assistant aux rencontres d'un groupe de soutien, si cette ressource leur convient. Ils peuvent y partager leur expérience, trouver de la compréhension et du soutien auprès d'autres parents qui ont aussi vécu la même épreuve. Ces rencontres peuvent les aider à avancer dans leur deuil.

\section{LA GROSSESSE SUIVANTE}

Dans les semaines qui suivent la perte d'un bébé, il y a souvent chez les parents une véritable obsession d'avoir un nouvel enfant le plus tôt possible. Certains pensent combler le vide, diminuer la souffrance, ramener la joie dans leur vie, remplacer un échec par un succès, réparer la blessure narcissique, prouver à l'entourage qu'ils sont capables de faire un bébé en vie, etc. Chaque fibre du corps de la mère était programmée pour prendre soin d'un bébé et le sentiment de vide et de manque peut prendre toute la place.

Certains parents choisissent de faire un autre bébé plutôt que de faire leur deuil. Mais la grossesse suivante ne permet pas de régler le deuil du bébé : il peut arriver que le deuil soit arrêté et qu'il se continue après l'accouchement, avec danger de dépression (Rousseau, 1988). Ou des éléments du deuil feront surface à nouveau durant la grossesse et viendront augmenter le stress des parents. Sans compter qu'en l'absence de détachement de l'enfant mort, les risques associés à l'enfant de remplacement sont grands.

Deux problèmes particuliers se retrouvent souvent durant la grossesse suivante:

- L'anxiété reliée à la peur que la tragédie se produise à nouveau. C'est non seulement difficile à vivre par le couple, mais l'anxiété provoque l'utilisation fréquente des services de santé. Durant la grossesse, il est important pour la femme de sentir que son médecin traitant la suit de plus près. Mais elle a aussi besoin de quelqu'un qu'elle peut appeler si elle est en état de panique. C'est pour cela que le service d'accompagnement de deuil continue à être disponible durant la grossesse suivante.

- La difficulté d'attachement au bébé suivant. Plusieurs parents ont de la difficulté à s'attacher au bébé à venir, de peur de le perdre et d'être encore blessé. Il est fréquent de voir les parents attendre pour apprendre la grossesse à l'entourage et pour préparer la chambre du bébé.

Si ce bébé est perdu lui aussi, non seulement la mère se sera privée de toutes les petites joies venant avec la grossesse, mais elle peut penser que son absence d'investissement envers ce bébé l'a empêché de naître vivant et ressentir beaucoup de culpabilité.

\section{L'ENFANT SUIVANT}

On peut penser qu'à la naissance d'un bébé vivant tous les problèmes sont réglés. Mais, selon Rousseau (1988), les écrits rapportent de nombreux cas de troubles psychiatriques chez les enfants (même devenus adultes) quand leur naissance suit la mort d'un autre enfant: psychose, troubles d'identité, d'identification sexuelle, culpabilité du survivant, toxicomanies, etc.
Si le deuil du bébé perdu n'est pas en voie de résolution et que la grossesse survient trop rapidement, les parents peuvent être trop perturbés pour répondre aux besoins du bébé.

De plus, le bébé mort est souvent idéalisé surtout s'il a vécu un peu. Le bébé actuel est comparé : jamais à son avantage. Si le bébé est difficile, il est facile de penser que celui qui est décédé aurait mieux répondu à leurs attentes. Donc, ce bébé déçoit et il devient plus difficile de s'y attacher. S'il y a difficulté d'attachement, l'enfant est très à risque d'être maltraité ou négligé.

Par contre, d'autres parents auront tendance à surprotéger ce bébé tellement précieux. Les parents veulent ce qu'il y a de mieux pour leur enfant. Mais ils ne savent pas que si l'enfant est surprotégé, il n'apprend pas à avoir confiance en lui et en la vie : il ressent l'anxiété de ses parents. La surprotection peut être aussi dommageable pour l'enfant que la négligence. Ce sont des points qui sont discutés avec les parents durant le suivi.

\section{LE SOUTIEN DES INTERVENANTS}

Il est difficile pour les membres de l'équipe de travailler avec des parents qui vivent beaucoup de souffrance. Avec l'expérience, une partie du stress est diminuée. Mais chaque parent étant unique, il est important qu'un intervenant puisse compter sur les autres membres de son équipe pour l'aider à résoudre les cas plus difficiles. Des rencontres mensuelles de l'équipe permettent de ventiler, de recevoir du soutien, d'acquérir des connaissances nouvelles, de discuter des cas. Ainsi, au Centre hospitalier Pierre-Boucher, nous avons la chance d'avoir un formateuraccompagnateur, un psychothérapeute d'approche analytique, monsieur Villemaire Paquin, qui vient bénévolement mettre son expérience à notre service. Sa participation nous aide considérablement à comprendre les difficultés rencontrées dans notre travail avec les parents. Il nous donne des pistes de réflexion pour favoriser le cheminement des parents et nous aide à conserver notre sérénité et notre engagement, malgré les difficultés de notre travail. Les membres de l'équipe servent de personnes-ressources dans l'hôpital. Ils transmettent aussi les commentaires positifs des parents sur les soins reçus, ce qui est valorisant pour les autres intervenants et les motive à s'impliquer auprès des parents nouvellement éprouvés.

Dans les conclusions de son étude sur le hardiness, comme facteur de ressource pour traverser un deuil périnatal, Lang (1992) souligne que cette composante personnelle peut être développée. Elle fait ressortir l'importance pour les parents de 
bénéficier d'interventions pertinentes de soutien. Le but de l'accompagnement de deuil, c'est d'offrir ce soutien : une oreille attentive, une présence réconfortante et disponible, une compréhension de ce que les parents vivent, un encouragement à exprimer leurs émotions pour les faire évoluer et une source de renseignements sur le deuil.

L'accompagnement de deuil périnatal est un bon outil de prévention en santé mentale. Le suivi de deuil permet d'éviter, ou de déceler tôt, plusieurs deuils pathologiques qui entraînent un dysfonctionnement de la personne et de la famille dont les enfants sont les premières victimes. De plus, en intervenant auprès de couples qui perdent quelqu'un de significatif, souvent pour la première fois dans leur vie, en les soutenant et au besoin en les guidant dans la façon saine de vivre un deuil, l'influence bénéfique s'étend aussi à leurs enfants qui apprendront correctement à faire leurs deuils et après eux leurs enfants aussi. D'où l'importance qu'il y ait une suite après le séjour au centre hospitalier et qu'on intervienne avant que le milieu n'ait influencé les parents et les ait encouragés à se refermer sur eux-mêmes et à faire un tabou de l'événement.

Sans compter que nos interventions, en donnant de l'importance à la perte, modifient la perception que l'entourage a de l'événement et sont donc susceptibles d'influencer positivement le soutien que les parents vont recevoir. À mesure que l'impact de la perte d'un bébé durant la grossesse ou peu après la naissance sera reconnu par la société, on peut espérer que les parents recevront un meilleur soutien de leur entourage et auront moins besoin d'un accompagnement professionnel.

Le suivi de deuil est un service très apprécié des parents. Le témoignage des intervenantes professionnelles du Centre hospitalier Pierre-Boucher confirme qu'il est gratifiant et encourageant pour elles de constater le cheminement des parents.

Un autre avantage appréciable a été constaté : lors des contacts avec les parents, les intervenantes reçoivent aussi des commentaires sur les interventions qui ont été faites lors de l'hospitalisation. On sait ainsi comment certaines pratiques, comme de prendre plusieurs photos du bébé ou la suggestion d'intégrer les autres enfants à l'événement, sont aidantes et appréciées par les parents, ce qui nous confirme que nous sommes dans la bonne voie et nous amène à nous améliorer constamment.

Quand on regarde tout ce que l'accompagnement de deuil périnatal peut apporter, on peut constater beaucoup de résultats positifs en quelques heures de soutien.

\section{Bibliographie}

BYDLOWSKI, M. (1997). La dette de vie: Itinéraire psychanalytique de la maternité, Paris, Presses universitaires de France.

DOHMEN, B. (1996). « Les ratés de la maternité », Psycorps, vol. 1 n 2 (automne).

FRÉCHETTE-PIPERNI, S. (1999). Deuil périnatal, Guide d'interventions pour les infirmières, Centre hospitalier Pierre-Boucher, Longueuil.

FRÉCHETTE-PIPERNI, S. (2001). « La perte d'un bébé et les autres enfants de la famille », Soins Pédiatrie-Puériculture, no 199 (mars/ avril), p. 31-36.

LANG, A. (2002). Testing of a Explanatory Model of Hardiness in Bereaved Couples Following the Death of Their Foetus / Infant, thèse doctorale, Université de Montréal.

LÉON, I. (1992). "Perinatal loss: A critique of current hospital practice», American Journal of Psychiatry, $\mathrm{n}^{\circ}$ 149, p. 1464-1472.

LINDEMANN, E. (1944). "Sympathologie and management of acute grief ", American Journal of Psychiatry, $\mathrm{n}^{\circ}$ 101, p. 141-148.

ROUSSEAU, P. (1997). Les dossiers de l'obstétrique, $\mathrm{n}^{\circ} 247$ (février), p. 4-6.

ROUSSEAU, P. (1988a). «Le deuil périnatal. Accompagnement de la famille. Prévention des répercussions chez les enfants ", Association Naître et Vivre.

ROUSSEAU, P. (1988b). «Le deuil périnatal. Psychopathologie et accompagnement», Journal of Gynecology \& Obstetric, $\mathrm{n}^{\circ} 17$, p. 285-294.

STROEBE, M.S., R.O. HANSSON, W. STROEBE et H. SCHUT (2001). Handbook of Bereavement Research, American Psychological Association, Washington, DC. 